\title{
Substitution of saturated with monounsaturated fat in a 4-week diet affects body weight and composition of overweight and obese men
}

\author{
L. S. Piers ${ }^{1 *}$, Karen Z. Walker ${ }^{2}$, Rachel M. Stoney ${ }^{3}$, Mario J. Soares ${ }^{4}$ and Kerin O’Dea ${ }^{1}$ \\ ${ }^{1}$ Menzies School of Health Research, Casuarina, Northern Territory, Australia \\ ${ }^{2}$ Nutrition and Dietetics, Department of Medicine, Monash University, Monash Medical Centre, Clayton, Victoria, \\ Australia \\ ${ }^{3}$ The Nutrition Department, The Alfred Hospital, Prahran, Victoria, Australia \\ ${ }^{4}$ Department of Nutrition, Dietetics and Food Science, Curtin University of Technology, School of Public Health, \\ Perth, Western Australia, Australia
}

(Received 1 August 2002 - Revised 11 April 2003 - Accepted 29 May 2003)

\begin{abstract}
A randomised crossover study of eight overweight or obese men (aged $24-49$ years, BMI $25 \cdot 5-31 \cdot 3 \mathrm{~kg} / \mathrm{m}^{2}$ ), who followed two diets for 4 weeks each, was performed to determine whether substitution of saturated fat with monounsaturated fat affects body weight and composition. Subjects were provided with all food and beverages as modules (selected ad libitum) of constant macronutrient composition, but differing energy content. The $\%$ total energy from saturated fat, monounsaturated fat and polyunsaturated fat was 24,13 and $3 \%$ respectively on the saturated fatty acid (SFA)-rich diet and 11, 22 and $7 \%$ respectively on the monounsaturated fatty acid (MUFA)-rich diet. MUFA accounted for about $80 \%$ of the unsaturated fats consumed on both diets. Body composition, blood pressure, energy expenditure (resting and postprandial metabolic rates, substrate oxidation rate, physical activity), serum lipids, the fatty acid profile of serum cholesteryl esters and plasma glucose and insulin concentrations were measured before and after each diet period. Significant $(P \leq 0 \cdot 05)$ differences in total cholesterol and the fatty acid composition of serum cholesteryl esters provided evidence of dietary adherence. The men had a lower weight $(-2.1$ (SE 0.4) $\mathrm{kg}, P=0.0015)$ and fat mass $(-2.6$ (SE 0.6) $\mathrm{kg}, P=0.0034)$ at the end of the MUFA-rich diet as compared with values at the end of the SFA-rich diet. No significant differences were detected in energy or fat intake, energy expenditure, substrate oxidation rates or self-reported physical activity. Substituting dietary saturated with unsaturated fat, predominantly MUFA, can induce a small but significant loss of body weight and fat mass without a significant change in total energy or fat intake.
\end{abstract}

Obesity: Dietary fat: Weight loss: Body composition: Energy expenditure

Despite their established health benefits, diets with a high monounsaturated fatty acid (MUFA) content are often not promoted due to concerns that their relatively high dietary total fat content might promote obesity (Garg, 1994; deLorgeril \& Salen, 2000), as fat per se not only forms highly palatable and energy-rich foods (Blundell \& MacDiarmid, 1997), but is also efficiently stored in the body (Stubbs, 1998). Although population and intervention studies have suggested the importance of high-fat diets in the promotion of obesity (Lissner \& Heitmann, 1995), a consensus establishing the relationship between a high fat intake and obesity has not yet been established (Bray \& Popkin, 1998; Willet, 2002). Recent evidence implicates saturated fatty acids (SFA), rather than the unsaturated fats, in the development of obesity, due to the greater propensity for SFA to be stored in adipose tissue rather than being oxidised (Storlien et al.
1998, 2001). Studies using indirect calorimetry in human volunteers have indicated that polyunsaturated fatty acids (PUFA) are better oxidised than SFA in both normal weight (Jones \& Schoeller, 1988) and obese men (Jones et al. 1992). Fewer studies, however, examine the fate of the nutritionally important MUFA. Jones et al. (1985), using stable isotope-labelled fatty acids of similar chain length, demonstrated that monounsaturated oleic acid $(18: 1)$ is more readily oxidised than saturated stearic acid $(18: 0)$ or polyunsaturated linoleic acid $(18: 2)$ (stearic:linoleic:oleic oxidation ratio $1 \cdot 0: 4 \cdot 5: 14 \cdot 0)$. We too have previously reported a greater postprandial fat oxidation rate $(P<0.05)$ in fourteen men after consumption of a breakfast meal rich in MUFA, as compared with a SFA-rich meal (Piers et al. 2002). Together, these results indicate that the predominance of certain fatty acids in a given dietary fat

Abbreviations: CE, cholesteryl ester; EE, energy expenditure; FM, fat mass; MUFA, monounsaturated fatty acid; PUFA, polyunsaturated fatty acid; SFA, saturated fatty acid.

* Corresponding author: Dr L. S. Piers, present address, Health Surveillance and Evaluation Section, Rural and Regional Health and Aged Care Services, Department of Human Services, Level 18, 120 Spencer Street, Melbourne, Victoria 3000, Australia, fax+61396374763, emailleonard.piers@ dhs.vic.gov.au 
influences its in vivo partitioning between oxidation and storage.

We, therefore, hypothesised that if a MUFA-rich diet were compared with a SFA-rich diet, the higher rate of fat oxidation on the MUFA-rich diet would result in reduced energy intake and/or the loss of body fat mass (FM). Reduced food intake would compensate for the greater amount of energy liberated from ingested food high in MUFA. Loss of FM would result if the rate of fat deposition declined while the rate of endogenous fat mobilisation remained stable. Furthermore, on a MUFArich diet, an enlarged body fat store would no longer be required to achieve high fat oxidation rates, as would be the case when poorly oxidisable SFA was consumed (Flatt, 1995).

To test these hypotheses, we invited all subjects from our previous acute study (Piers et al. 2002) to participate in a longer intervention study examining the influence of the type of dietary fat on energy intake, body weight and composition. Subjects were required to consume two diets of constant macronutrient composition and with a fat content reflecting that of an average western diet (about $40 \%$ total energy), but differing in fatty acid composition (one being rich in SFA and the other in MUFA) in a randomised crossover study. Each diet was consumed for 4 weeks.

\section{Subjects and methods}

\section{Subjects}

Subjects for the present study were recruited from participants of our earlier acute study investigating fat oxidation rates by indirect calorimetry (Piers et al. 2002). As the results of the earlier study were not available at that time, subjects were only told that we were interested in seeing if the consumption of two special diets, for 4 weeks each, resulted in any change in fat oxidation rates in the longer term. Subjects remained unaware that a change in body weight or composition might be expected. Eight of the fourteen volunteers agreed to participate in the intervention study and the remaining men declined due to the time commitment required. The eight male subjects (seven of European and one of South Asian ancestry) were aged between 24 and 49 years and were resident in Melbourne, Victoria, Australia. Inclusion criteria were as in the previous study (Piers et al. 2002) including: (1) absence of clinical signs or symptoms of chronic disease; (2) weight stability ( $\pm 2 \mathrm{~kg}$ for preceding 12 months); (3) not on medication affecting body composition; (4) BMI within the range $25-32 \mathrm{~kg} / \mathrm{m}^{2}$; (5) resting diastolic blood pressure $<90 \mathrm{mmHg}$.

\section{Study design}

Subjects consumed two high-fat diets (40\% energy) of fixed macronutrient composition. The diets were designed to differ only in the type of fat provided, one diet predominantly containing MUFA and the other predominantly containing SFA. In order to determine whether total energy intake varied with the type of dietary fat consumed, each diet was administered in a modular form; this kept the proportion of macronutrients constant while permitting ad libitum energy intake. The order of administration of the diets was randomly assigned and there was no intervening washout period. Anthropometric measurements, body composition and blood pressure measurements were made initially and at the end of each diet period. Resting and postprandial metabolic rate were also measured in response to an isoenergetic test meal of constant macronutrient composition. However, the type of fat in the test meal reflected the type of fat that had been predominantly consumed in the associated diet period. Fasting and $2 \mathrm{~h}$ postprandial venous blood samples were collected on these occasions. In addition, subjects were required to maintain a self-reported physical activity diary daily for the entire duration of the two diet periods.

\section{Diets}

Sources of fat for the SFA-rich diet were milk, butter, cream, cheese and fatty meat, while fat in the MUFArich diet was provided from olive oil, nuts and avocados. Each diet comprised forty modules, paired between diets for similar energy, protein, carbohydrate, total fat and fibre content, and with the exception of the fat source, based on largely similar foods. Each diet contained ten breakfast modules $(0 \cdot 7-2 \cdot 2 \mathrm{MJ})$, ten lunch modules $(0 \cdot 9-4 \cdot 1 \mathrm{MJ})$, ten dinner modules $(3 \cdot 1-5 \cdot 3 \mathrm{MJ})$ and ten snack modules $(1 \cdot 0-4 \cdot 2 \mathrm{MJ})$. The forty modules provided for the SFA-rich diet contained on average (\% energy): fat 40.3 (SD 0.8), SFA 24.4 (SD 2.6), MUFA 12.5 (SD 1.5 ), carbohydrate 41.9 (SD 3.7). The forty modules provided for the MUFA-rich diet were designed to contain on average (\% energy): fat $40 \cdot 1$ (SD 1.1), SFA 11.0 (SD 0.9), MUFA 22.3 (SD 1.7), carbohydrate 42.2 (SD 3.6). An exception was two paired snack modules containing wine or beer (\% energy): alcohol 19.2, fat 40.7, carbohydrate 27.2. The MUFA-rich diet modules provided slightly more dietary fibre than the SFA-rich diet modules (3.4 (SD 1.8) v. 2.8 (SD 1.9) g/MJ respectively). Examples of the modules are provided in Table 1 . The macronutrient composition of each module, and the module recipe can be obtained by contacting the corresponding author (L. S. P.).

Each week, each subject chose freely from the module menu and could consume their chosen modules in any order and in any quantity, each day. Modules were always provided in excess of intake requirements. Subjects were instructed that once the consumption of a module was started, the entire module had to be consumed. No food other than that provided was allowed. All food was prepared under the supervision of a research dietitian at The Kingston Centre (Cheltenham, Victoria, Australia), a hospital for geriatric patients with large food service facilities. Modules were given to subjects mainly in a ready-to-eat form. Some modules required reheating or simple cooking. Detailed storage and preparation instructions were provided for each module. Each subject completed, on a daily basis, a record detailing all modules consumed each day, over the four weeks of each diet. 
Table 1. Examples of modules provided in the saturated fatty acid-rich and monounsaturated fatty acid-rich test diets

\begin{tabular}{lll}
\hline Module & \multicolumn{1}{c}{ SFA-rich modules } & MUFA-rich modules \\
\hline 1 & Natural muesli with creamy milk & Toasted muesli with skimmed milk \\
2 & Tomatoes on buttered toast & Tomatoes fried in olive oil, toast \\
4 & Poached egg, toast and butter, orange juice & Egg fried in olive oil, toast, skimmed milk, orange juice \\
5 & Cornflakes and creamy milk & Cornflakes, cashews and skimmed milk \\
14 & Muffins, butter and cottage cheese & Muffins, high monounsaturated fat margarine, cottage cheese \\
15 & Vegetable soup and toast with butter & Soup made with vegetables fried in olive oil, toast \\
22 & Rump steak stir-fried with celery and & Rump steak stir-fried with celery, almonds \\
& butter, boiled rice & and olive oil, boiled rice \\
24 & Fish cooked in butter, peas, carrots, & Fish fried in olive oil, peas, \\
& potato, oranges & carrots, potato, oranges \\
27 & Roast chicken with carrots and beans, & Roast chicken with carrots and beans, \\
& baked potato with butter and soured & baked potato with high-monounsaturated fat margarine, \\
& cream, canned apricots with cream & canned apricots \\
31 & Cheesecake and ice cream, a glass & Fruit cake, ice cream, almonds, and \\
33 & of creamy milk and a banana & a glass of skimmed milk \\
37 & Chocolate biscuit with glass of creamy milk & Ginger biscuit, almonds and a glass of skimmed milk \\
& One glass of white wine, cheese and sultanas & One glass of white wine, almonds and sultanas
\end{tabular}

SFA, saturated fatty acid; MUFA, monounsaturated fatty acid.

\section{Breakfast meals used for postprandial studies}

The SFA-rich breakfast comprised $92 \mathrm{~g}$ natural Swiss muesli (Uncle Toby's; Wahgunyah, Victoria, Australia), $57 \mathrm{~g}$ cream (Bulla, Regal Cream Products; Colac, Victoria, Australia) and $275 \mathrm{~g}$ skimmed milk (Australian Milk Marketing; Abbotsford, Victoria, Australia). The MUFA-rich breakfast consisted of $96 \mathrm{~g}$ muesli baked with $20 \mathrm{~g}$ extra virgin olive oil (Bertolli; Preston, Victoria, Australia) and served with $285 \mathrm{~g}$ skimmed milk. Both breakfast meals, which have been used previously (Piers et al. 2002), provided $2.5 \mathrm{MJ}$ energy: $15 \%$ as protein, $43 \%$ as fat and $42 \%$ as carbohydrate. The fibre content of the SFA-rich breakfast was $12.0 \mathrm{~g}$, while that of the MUFA-rich breakfast was $11.5 \mathrm{~g}$. The SFA-rich breakfast contained $4.1 \mathrm{~g}$ PUFA, $8.8 \mathrm{~g}$ MUFA and $16.1 \mathrm{~g}$ SFA, while the MUFArich breakfast contained 5.6 g PUFA, $18.6 \mathrm{~g}$ MUFA and $4.9 \mathrm{~g}$ SFA, as determined from Australian food composition tables (NUTTAB95; English \& Lewis, 1991).

\section{Diet assessment questionnaire}

At the end of each diet, subjects were given a questionnaire regarding their assessment of diet quality. For each question, they were asked to indicate their response by marking a cross on an unmarked $100 \mathrm{~mm}$ line extending between two extreme ratings $(0,100)$ and the length to the mark was then measured with a ruler. The questions were: (1) How do you rate this diet overall? (2) How did this diet satisfy your hunger? (3) How do you describe the taste of this diet? (4) How energetic did you feel while on this diet?

\section{Anthropometry}

Standing height was measured using a wall-fixed stadiometer and recorded to the nearest $1 \mathrm{~mm}$. Body weight was measured immediately after voiding and after an overnight fast. Subjects wore light indoor clothing and no shoes. The measurement was taken on a beam balance and was recorded to the nearest $100 \mathrm{~g}$. Waist and hip circumferences were measured as described by Callaway et al. (1988).

\section{Body composition}

Dual-energy X-ray absorptiometry whole-body scans were performed as described by Piers et al. (2002). Total and regional body composition was determined with Lunar software (Lunar software version 1.3; Madison, WI, USA) used in the extended research mode to provide data on whole-body and regional (limbs and trunk) lean tissue mass and FM (g).

\section{Resting and postprandial metabolic rate}

Resting and postprandial metabolic rate was measured by indirect calorimetry using a Deltatrac II metabolic monitor (Datex Instrumentarium Corp., Helsinki, Finland), an opencircuit ventilated canopy measurement system. The measurement was conducted under standardised conditions using the same measurement protocol as employed in our previous study (Piers et al. 2002).

\section{Substrate oxidation rates}

Whole-body substrate oxidation rates were calculated using measures of $\mathrm{O}_{2}$ consumption, $\mathrm{CO}_{2}$ production, total urinary $\mathrm{N}$ excretion and the equations of Ben-Porat et al. (1983), with the assumption that $\mathrm{N}$ excretion was steady over the duration of urine collection (Elia \& Livesey, 1988; Livesey \& Elia, 1988).

\section{Blood pressure and pulse rate}

Systolic and diastolic blood pressure and pulse rate were measured before and after each diet period under standardised conditions (after an overnight fast, in the postabsorptive state, after having rested in the supine position for at least $1 \mathrm{~h}$ ) using a Dinamap portable adult vital signs monitor (model 100; Critikon, Tampa, FL, USA). 


\section{Physical activity assessment}

All subjects were asked to maintain a daily physical activity diary. The diary consisted of four 15 min blocks for each hour of the day from 06.00 to 23.00 hours. It was assumed that subjects slept between 23.00 and 06.00 hours, but they were asked to indicate their actual waking and sleeping times when there was any deviation from this pattern. Subjects were asked to write down the nature of the activity predominantly performed during each $15 \mathrm{~min}$ block. These activities were then coded depending upon the type and intensity of activity. The total number of $15 \mathrm{~min}$ blocks at each activity level was determined. Energy expenditure (EE) at each level of activity was obtained by multiplying the measured resting metabolic rate (kJ per $15 \mathrm{~min}$ ) by an activity factor obtained from the literature (Food and Agriculture Organization/World Health Organization/United Nations University, 1985), appropriate for the type of activity being performed, and by the number of $15 \mathrm{~min}$ blocks for which the activity was performed during the day. EE during sleep was assumed to be equal to resting metabolic rate measured at the end of each diet period. The sum of the EE associated with the levels of activity over $24 \mathrm{~h}$ gave total EE $(\mathrm{kJ} / \mathrm{d})$. Physical activity levels were calculated by dividing the total $\mathrm{EE}(\mathrm{kJ} / \mathrm{d})$ obtained from the self-reported activity diaries by measured resting metabolic rate $(\mathrm{kJ} / \mathrm{d})$.

\section{Blood samples}

Fasting and $2 \mathrm{~h}$ postprandial (following the standard breakfast meal) blood samples $(10 \mathrm{ml})$ were collected from all subjects before and at the end of each diet period. The samples were centrifuged at $2000 \mathrm{~g}$ for $15 \mathrm{~min}$ before plasma was separated and stored at $-70^{\circ} \mathrm{C}$. Plasma glucose, insulin concentrations and the lipid profile were measured in the fasting sample as described by Piers et al. (2002).

\section{Serum fatty acid analysis}

Fatty acid analysis was carried out at the Child Nutrition Research Centre, Flinders Medical Centre, South Australia, Australia. Lipids were extracted from serum samples with chloroform-methanol (Bligh \& Dyer, 1959). Serum phospholipids and cholesteryl esters (CE) were separated by TLC and evaporated to dryness under $\mathrm{N}_{2}$. The samples were methylated in $\mathrm{H}_{2} \mathrm{SO}_{4}(10 \mathrm{ml} / 1$ methanol $)$ at $70^{\circ} \mathrm{C}$ for $3 \mathrm{~h}$. When cooled, methyl esters were extracted into $n$-heptane and transferred to vials containing anhydrous $\mathrm{Na}_{2} \mathrm{SO}_{4}$ as the dehydrating agent. Fatty acid methyl esters were separated and quantified using a Hewlett-Packard 6890 GC (Hewlett-Packard, Palo Alto, CA, USA) equipped with a $50 \mathrm{~mm}$ capillary column (internal diameter $0.33 \mathrm{~mm})$ coated with BPX-70 $(0.25 \mu \mathrm{m}$ film thickness; SGE Pty Ltd, Victoria, Australia). The injector temperature was set at $250^{\circ} \mathrm{C}$ while the detector (flame ionisation) temperature was $300^{\circ} \mathrm{C}$. The oven temperature commenced at $140^{\circ} \mathrm{C}$ and was programmed to rise at $5^{\circ} \mathrm{C}$ per min to $220^{\circ} \mathrm{C}$. The carrier gas $\mathrm{He}$ was used at a velocity of
$350 \mathrm{~mm} / \mathrm{s}$. Fatty acid methyl esters were identified based on retention time relative to authentic lipid standards (Nuchek Prep Inc., Elysian, MN, USA). Between-run variations of the same sample for major fatty acid peaks $(>2 \%)$ were $<1 \%$.

\section{Ethics}

All subjects gave written informed consent to participate in the study. The Standing Committee on Ethics in Research on Humans, Monash University, approved the experimental protocol.

\section{Statistical analysis}

Data were analysed using the STATA Statistical Software (Release 7, College Station, TX, USA) statistical software package. All results are presented as mean values and standard deviations unless otherwise stated. The outcome variables of interest were body weight and FM. The covariates included energy intake, total fat intake, total EE associated with physical activity and physical activity level. The change in a variable of interest associated with a particular diet and the difference between the changes in a variable of interest between the two diet periods, in the same subject, were obtained as follows:

$$
\text { change in variable }=\text { measure after diet }
$$

$$
\text { - measure before diet }
$$

and

difference in change $=$ change on SFA-rich diet

$$
\text { - change on MUFA-rich diet. }
$$

Where no pre-intervention measures were made, measures made at the end of each diet period were compared. Pearson correlation coefficients were computed to examine relationships between variables of interest. The difference between the changes in the variables of interest, or the difference in variables at the end of each diet period, were analysed using ANOVA for the $2 \times 2$ crossover study design. This is similar to repeated measures, except that the treatment factor (effects of the two diets in question) was assigned across the time periods. Each subject received both treatments in a particular sequence (sequence in which the diets were administered: SF-rich diet $\rightarrow$ MUFA-rich diet $=1$, MUFA-rich diet $\rightarrow$ SFA-rich diet $=2$ ). Within each subject, a period effect (the temporal order in which the diets were administered to each individual in each sequence) was also assessed. It was not possible to separate the sequence effect from any carry-over effect, as only two time periods were considered (Yandell, 1997). A significant $(P \leq 0 \cdot 05)$ sequence effect indicates that caution is required in interpreting treatment effects. When this occurred, we went on to compare the effects by treatment differences between diets in the first study period only, using an unpaired $t$ test as has been recommended (Yandell, 1997). A paired $t$ test was used to 
compare paired data within individuals. Regression analysis with robust variance estimates (Stata Reference Manual: Version 7, 2001; STATA Corporation) was used to test for any treatment, sequence and period effects, after adjusting for confounding factors.

\section{Results}

\section{Dietary intake}

Dietary intake data for the two diet periods are presented in Table 2. On the SFA-rich diet, men consumed $24 \%$ energy as SFA, $3 \%$ energy as PUFA and $13 \%$ energy as MUFA, whereas on the MUFA-rich diet the men consumed $11 \%$ energy as SFA, $6 \%$ energy as PUFA and $23 \%$ energy as MUFA. Proportionately, MUFA accounted for approximately $80 \%$ of the unsaturated fatty acids consumed on both diets. Although subjects had been asked to eat the dietary modules ad libitum, results indicated no significant differences between the SFA- and MUFA-rich diets in total energy $(P=0 \cdot 16)$, fat $(P=0 \cdot 17)$ or carbohydrate $(P=0.37)$ intake (ANOVA). However, in the case of total energy and total fat intake, a significant sequence effect was apparent $(P=0.05$ and $P=0.04$ respectively). Consequently, differences in these variables were examined for the first diet period alone, a procedure recommended when a significant sequence effect is detected (Yandell, 1997). This analysis (using an unpaired $t$ test) showed that during the first diet period, those individuals following an SFA-rich diet had consumed significantly more (14.3 (SD 2.3) MJ/d, $n 4$ ) energy than those individuals following the MUFA-rich diet (10.6 (SD 0.5) MJ/d, $n 4 ; P<0.05)$. The best explanation for this difference would appear to be the greater amount of heavy physical activity by the four individuals receiving the SFA-rich diet in the first diet period (214 (SD 250) kJ/d) compared with those on the MUFArich diet (20 (SD 40) $\mathrm{kJ} / \mathrm{d} ; P=0.22$, trend only), a 'between-subject' rather than 'within-subject' difference. A significant correlation was also evident between physical activity level and total energy intake in those on the SFArich diet $(n 4, r 0.99, P=0.002)$, but not in those on the MUFA-rich diet $(P=0 \cdot 10)$.

In accordance with the study design, there were statistically significant differences in the consumption of different types of fat, intakes of SFA were substantially greater $(P<0.00005)$, and MUFA $(P<0.00005)$ and PUFA $(P=0.0002)$ intakes substantially less, on the SFA-rich diet. However, once again a significant sequence effect $(P=0.04)$ was evident in the ANOVA of SFA intake. Subsequent regression analysis of SFA intakes, with physical activity level as a covariate, showed a difference in SFA intakes between diets $(P<0.0005)$; however, the sequence effect was no longer significant $(P=0 \cdot 07)$. Although subjects consumed less dietary fibre on the SFA-rich diet $(P=0.02)$, once again there was a significant sequence effect $(P=0.003)$. An independent $t$ test of the fibre intake during the first dietary period showed that subjects $(n 4)$ on the SFA-rich diet consumed a significantly greater amount of fibre (42.3 (SD 1.8) g/d) compared with subjects $(n 4)$ on the MUFA-rich diet $(34.5$ (SD 1.6) $\mathrm{g} / \mathrm{d}$; $P=0 \cdot 02)$. There was no significant difference between diets in alcohol intake $(P=0 \cdot 55)$.

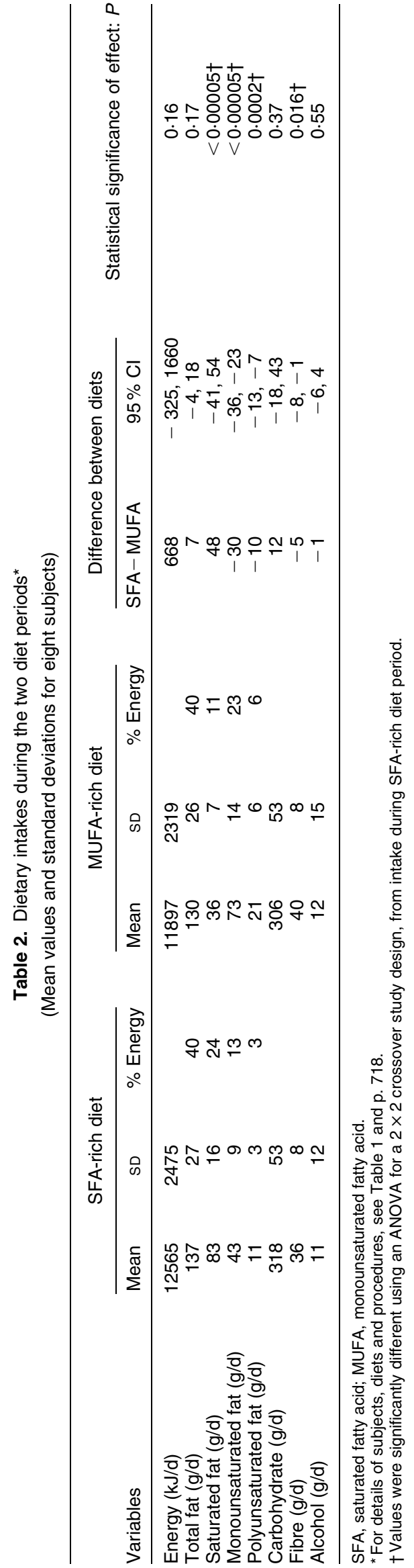




\section{Diet ratings}

Subjects indicated (scale $0-100)$ that they felt more physically energetic on the MUFA-rich diet (81 (SD 18)) than on the SFA-rich diet (55 (SD 24)), a difference that was statistically significant (ANOVA, $P=0 \cdot 01$ ). They also tended to prefer the MUFA-rich diet (78 (SD 12)) to the SFArich diet (60 (SD 29)), although this difference fell short of statistical significance $(P=0 \cdot 09)$. Otherwise, subjects found the two diets comparable in terms of satiety (SFArich diet 80 (SD 21) v. MUFA-rich diet 89 (SD 15); $P=0.32$ ) and taste (SFA-rich diet 66 (SD 23) $v$. MUFArich diet 69 (SD 17); $P=0 \cdot 80$ ).

\section{Anthropometry and body composition}

All subjects had a BMI $>25 \mathrm{~kg} / \mathrm{m}^{2}$. Six of the eight subjects had waist circumferences $>0.99 \mathrm{~m}$. Although there was no significant difference in the change in waist circumference $(P=0.15)$ or change in hip circumference $(P=0.73)$ during the two diet periods, the difference in the change in waist:hip ratio $(0.03)$ was of borderline statistical significance $(P=0 \cdot 05$, Table 3$)$.

Body weight and composition, and the absolute change on the two diets, are presented in Table 3. ANOVA showed significantly higher body weight after the SFAthan the MUFA-rich diet $(P=0 \cdot 0015)$. FM $(P=0 \cdot 0037)$, $\%$ body fat $(P=0.03)$, trunk FM $(P=0.01)$ and limb FM $(P=0.03)$ (Fig. 1) were also significantly greater after the SFA-rich diet. However, a significant sequence effect $(P=0.03)$ was evident in the ANOVA of the change in limb FM. Once again, an unpaired $t$ test of the difference in the change in limb FM in the first dietary period only was carried out (Yandell, 1997). The change was significantly greater in the subjects on the SFA-rich diet $(0.7$ (SD 0.6) kg, $n$ 4) compared with those on the MUFA-rich diet $(-0.9$ (SD 0.5) $\mathrm{kg}, n 4 ; P=0.005)$. No significant difference was found in the change in lean tissue mass $(P=0.20)$ (Fig. 1). FM measured at the end of the SFA-rich diet was found to remain significantly greater than that measured at the end of the MUFA-rich diet after adjusting for energy intake $(2.7$ (robust SE 0.7$) \mathrm{kg}, P=0.007$ ), total fat intake $(2.7$ (robust SE 0.7$) \mathrm{kg}, P=0.007)$, fibre intake ( 2.3 (robust SE 0.8$) \mathrm{kg}, P=0 \cdot 022$ ), self-reported total $\mathrm{EE}$ ( 2.7 (robust SE 0.6$) \mathrm{kg}, P=0.004$ ) or physical activity level (2.7 (robust SE 0.6) $\mathrm{kg}, P=0.004)$.

\section{Energy expenditure}

No significant differences (ANOVA, $P>0.05$ ) between diets were evident in the change in resting metabolic rate $(P=0.93)$ or change in diet-induced thermogenesis expressed in absolute terms $(P=0.54)$, as \% energy content in the meal $(P=0.55)$ or as change in post-meal total energy output $(P=0 \cdot 44)$.

\section{Total urinary nitrogen excretion and substrate oxidation rates}

There were no significant differences on ANOVA of the change in total urinary $\mathrm{N}$ excretion $(P=0 \cdot 80)$, protein oxidation rate $(P=0.80)$, fasting fat oxidation rate $(P=0.98)$, postprandial fat oxidation rate $(P=0.98)$, fasting carbohydrate oxidation rate $(P=0.97)$ or postprandial carbohydrate oxidation rate $(P=0.57)$ between the two diet periods (Fig. 2). The change in FM was not significantly correlated with postprandial fat oxidation rates on either the MUFA-rich $(r-0.25, P=0.56)$ or SFA-rich ( $r 0 \cdot 17, P=0.70$ ) diets. However, it tended to be inversely correlated with postprandial carbohydrate oxidation rates on the MUFA-rich $(r-0.65, P=0.08)$, but not on the SFA-rich $(r 0.20, P=0.64)$ diet.

\section{Blood pressure and pulse rates}

Table 3 shows the effect of diet on blood pressure and pulse rate. The difference in change in diastolic blood pressure $(P=0.08)$ and mean arterial pressure $(7.1 \quad(95 \%$ CI $-0.1,14.4) \mathrm{mmHg} ; P=0.05)$ were of borderline significance (ANOVA). No significant difference was found in the change in systolic blood pressure $(P=0.13)$ or in pulse rate $(P=0.92)$.

\section{Lipid profile, glucose and insulin concentrations}

Lipid profile, glucose and insulin concentrations, fatty acid composition of serum CE are presented in Table 4. There was a significantly greater change in total cholesterol concentration on the SFA-rich as compared with the MUFA-rich $(P=0.004)$ diet. However, the difference in the change in LDL-cholesterol was just short of statistical significance $(1 \cdot 1 \mathrm{mmol} / \mathrm{l}, P=0 \cdot 07)$. No significant differences were observed in the change in other lipid fractions, or in fasting glucose or insulin concentrations.

\section{Fatty acid profile in cholesteryl esters}

The absolute changes in all classes of fat found in plasma $\mathrm{CE}$ are presented in Table 4. Total SFA in CE $(\mathrm{g} / 100 \mathrm{~g}$ total fatty acids) was significantly greater on the SFA-rich as compared with the MUFA-rich $(P=0.0003)$ diet. However, as a significant sequence effect was observed, an unpaired $t$ test was performed to compare the subjects on the SFA-rich diet $v$. those on the MUFA-rich diet in the first diet period only (Yandell, 1997). This indicated a significantly $(P=0.02)$ greater change in percentage SFA in $\mathrm{CE}$ had occurred in the four subjects on the SFA-rich diet (2.5 (SD 1.1) g/100 g total fatty acids) as compared with the four subjects on the MUFA-rich diet $(-0.8$ (SD 0.8) $\mathrm{g} / 100 \mathrm{~g}$ total fatty acids). Total MUFA in CE $(P=0.04)$, but not oleic acid $(18: 1 n-9)$ content in $\mathrm{CE}(P=0 \cdot 61)$, was greater on the SFA-rich diet. Total $n-6$ PUFA in CE was lower on the SFA-rich diet $(P=0 \cdot 0001)$, while total $n-3$ PUFA $(P=0.0008)$ was higher. However, ANOVA indicated a significant $(P<0.05)$ sequence effect occurring with both $n-6$ and $n-3$ PUFA content. Analysis of the change in $n-6$ PUFA in CE on the two diets, with an independent $t$ test in the first study period, showed a decrease in the subjects on the SFA-rich diet $(-6 \cdot 1$ (SD 2.2) g/ $100 \mathrm{~g}$ total fatty acids, $n$ 4) and an increase in subjects on the MUFA-rich diet $(0.9$ (SD 1.1) $\mathrm{g} / 100 \mathrm{~g}$ total fatty acids, $n 4)$. The change in total $n$-6 PUFA in CE was 
Type of dietary fat influences body composition

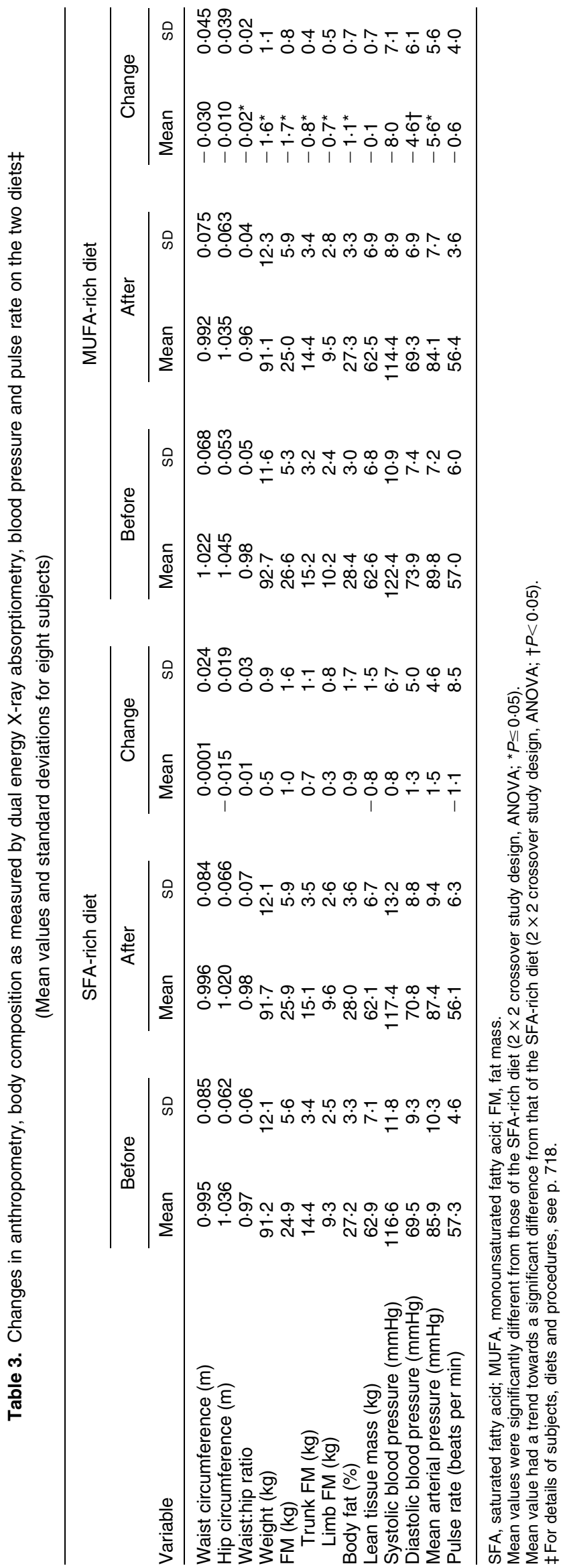




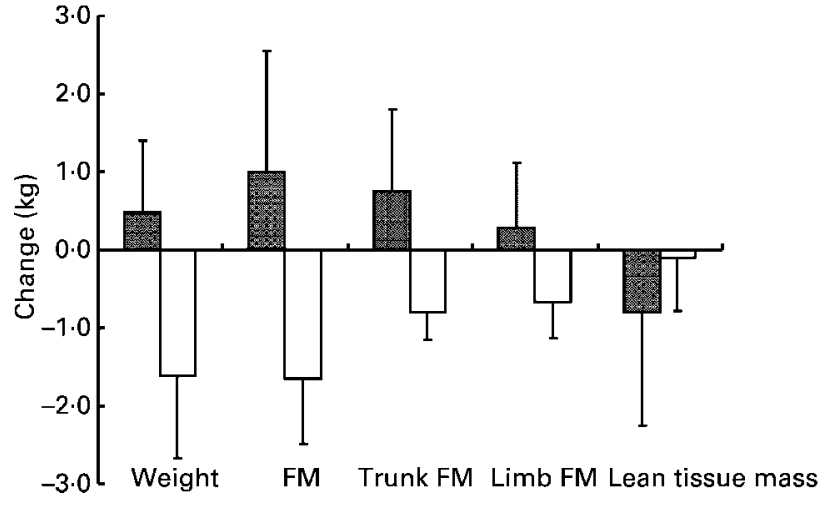

Fig. 1. Effect of saturated fatty acid-rich ( $\square$ ) and monunsaturated fatty acid-rich ( $\square$ ) diets on changes in body weight, fat mass (FM), trunk FM, limb FM and lean tissue mass. Values are means with standard deviations shown by vertical bars $(n 8)$. For details of subjects, diets and procedures, see Tables 1 and 2 and p. 718 .

predominantly due to a change in linoleic acid $(18: 2 n-6)$ $(P=0 \cdot 001)$

\section{Physical activity}

ANOVA of self-reported physical activity diaries indicated no significant difference in total EE between diets (SFArich diet $10.1(95 \%$ CI 9.2, 11.0) $v$. MUFA-rich diet 9.7 (95\% CI 8.9, 10.6) MJ/d; $P=0.40$ ). There were no significant differences in self-reported $\mathrm{EE}$ associated with sleep $(P=0 \cdot 71)$, sitting $(P=0 \cdot 42)$ and light $(P=0 \cdot 36)$, moderate

(A)
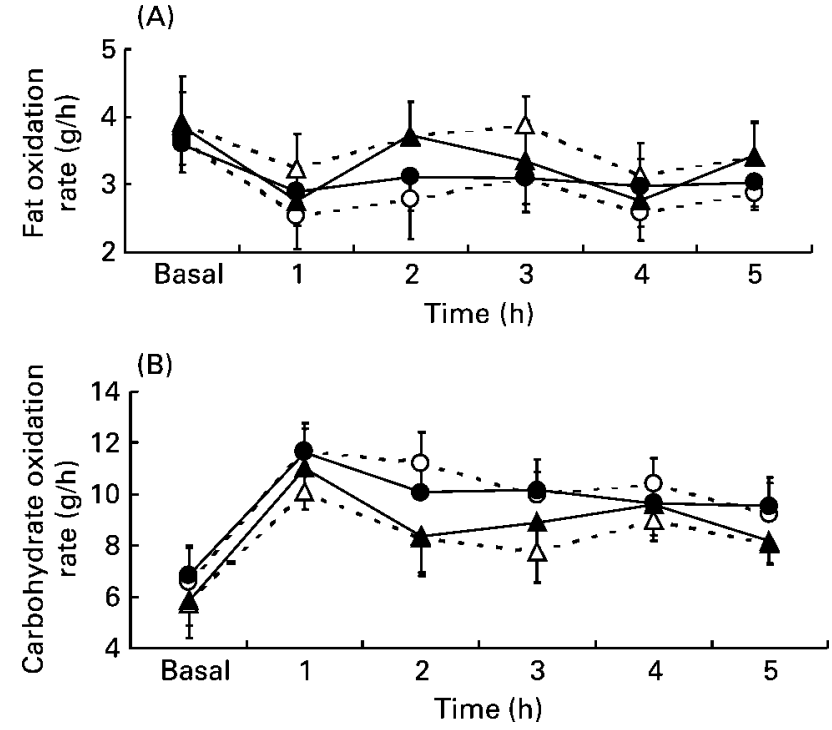

Fig. 2. Fat $(A)$ and carbohydrate oxidation rates $(B)$ measured in the basal state and following breakfast meals that differed only in the type of fat, one containing predominantly saturated fat and the other monounsaturated fat. The measurements were made prior to and at the end of two diet periods during which the macronutrient composition of the diet was held constant, but the type of fat was varied so that it was rich in saturated fatty acids (SFA) in one diet period and monounsaturated fatty acids (MUFA) during the other.-O-, Before SFA-rich diet; $-\Delta-$, before the MUFA-rich diet; -0-, after the SFA-rich diet; $\mathbf{- \Lambda}-$, after the MUFA-rich diet. Values are means with standard deviations shown by vertical bars ( $n$ 8). For details of subjects, diets and procedures, see Tables 1 and 2 and p. 718 .
$(P=0 \cdot 11) \quad$ or heavy physical activity $(P=0.99)$ (ANOVA). There was also no significant difference in physical activity level between the two diet periods $(P=0 \cdot 80)$.

\section{Discussion}

Overall, subjects gained FM (1.0 (SD 1.6) $\mathrm{kg}$ ) during the SFA-rich diet and lost FM ( -1.7 (SD 0.8) $\mathrm{kg}$ ) during the MUFA-rich diet (Table 3 ). These changes in body composition were observed despite the absence of statistically significant differences in total energy or fat intake during the two diet periods (Table 2). The changes in lean tissue mass in both diet periods were small and did not significantly affect the resting metabolic rate. Men on the SFArich diet gained FM predominantly on the trunk $(0.7$ (SD $1.1) \mathrm{kg}$ ) rather than on the limbs $(0.3$ (SD 0.8$) \mathrm{kg})$. In contrast, on the MUFA-rich diet, similar amounts of body fat were lost both from the trunk $(-0.8(\mathrm{SD} 0.4) \mathrm{kg})$ and from the limbs $(-0.7$ (SD 0.5) $\mathrm{kg}$ ) (Fig. 1), a finding that is in accordance with our previous study (Walker et al. 1996).

The higher FM $(+2.64 \mathrm{~kg})$ at the end of the SFA-rich diet as compared with the MUFA-rich diet, when expressed as energy amounted to $82.8 \mathrm{MJ}$ over 4 weeks, calculated at $31.4 \mathrm{~kJ} / \mathrm{g}$ fat (Berrino et al. 2001). Differences in energy intake and physical activity data on the two diets explain only part of this difference. We can attribute $46.5 \mathrm{MJ}$ of this difference to a greater energy intake on the SFA-rich diet (the upper limit of the $95 \% \mathrm{CI}$ in the mean difference in energy intake: $1660 \mathrm{~kJ} / \mathrm{d}$ for 4 weeks). A further $14 \mathrm{MJ}$ can be attributed to a subtle increase in physical activity on the MUFA-rich diet (the difference between upper limit of the $95 \%$ CI of total EE reported for the MUFA-rich diet and the mean total EE reported for the SFA-rich diet: 10.6 v. $10.1 \mathrm{MJ} / \mathrm{d}$ for 4 weeks respectively). There is some subjective evidence for this, as questionnaires given to the subjects at the end of each diet period indicated that subjects felt 'more energetic' on the MUFA-rich diet $(P=0.01)$, which is consistent with one of our earlier studies (Walker et al. 1995). The remaining difference can be explained on the basis of our previous results (Piers et al. 2002), relating to differences in fat oxidation rates between SFA-rich and MUFA-rich meals. In that study, we proposed that an individual whose energy intake was $10.0 \mathrm{MJ} / \mathrm{d}$ might oxidise an extra $670 \mathrm{~kJ} / \mathrm{d}$ by consuming MUFA rather than SFA. In the current study, the observed mean energy intake on the MUFA-rich diet was $11.9 \mathrm{MJ} / \mathrm{d}$. Extrapolating, an extra $797 \mathrm{~kJ} / \mathrm{d}$ might be burnt on the MUFA-rich diet, or $22.3 \mathrm{MJ}$ over 4 weeks, which is the energy difference otherwise unaccounted for.

The observed changes in body weight and body fat were accompanied by trends towards reduced waist:hip ratio and blood pressure (diastolic and mean arterial pressure) on the MUFA-rich diet (Table 4), indicative of physiological significance. It is well established that weight gain or loss, over as short a period as 1-2 months, is accompanied by an associated change in blood pressure (McCarron \& Reusser, 1996). In addition, the waist:hip ratio is positively and independently correlated with systolic arterial pressure in male hypertensive patients (Raison et al. 1992). 
Table 4. Changes in serum lipids, fatty acid profile of cholesteryl esters and plasma glucose and insulin concentrations on the two diets (Mean values and standard deviations for eight subjects)

\begin{tabular}{|c|c|c|c|c|c|c|c|c|c|c|c|c|}
\hline & \multicolumn{6}{|c|}{ SFA-rich diet } & \multicolumn{6}{|c|}{ MUFA-rich diet } \\
\hline & \multicolumn{2}{|c|}{ Before } & \multicolumn{2}{|c|}{ After } & \multicolumn{2}{|c|}{ Change } & \multicolumn{2}{|c|}{ Before } & \multicolumn{2}{|c|}{ After } & \multicolumn{2}{|c|}{ Change } \\
\hline & Mean & SD & Mean & SD & Mean & SD & Mean & SD & Mean & SD & Mean & SD \\
\hline \multicolumn{13}{|l|}{ Serum lipids } \\
\hline Total cholesterol $(\mathrm{mmol} / \mathrm{l})$ & 4.8 & 0.5 & 5.4 & 0.6 & 0.60 & 0.70 & $5 \cdot 3$ & 0.6 & 4.6 & 0.5 & $-0.76^{\star}$ & 0.56 \\
\hline Triacylglycerol $(\mathrm{mmol} / \mathrm{l})$ & 1.6 & 1.2 & $1 \cdot 8$ & 1.4 & -0.18 & $1 \cdot 13$ & 1.4 & 0.5 & 1.6 & 0.7 & 0.19 & 0.65 \\
\hline LDL-cholesterol (mmol//) & $3 \cdot 0$ & $0 . \overline{4}$ & 3.5 & 0.6 & 0.44 & 0.82 & 3.4 & 0.5 & $2 \cdot 8$ & 0.4 & $-0.56 \dagger$ & 0.43 \\
\hline HDL-cholesterol (mmol/) & 1.0 & 0.3 & 1.0 & 0.1 & 0.01 & 0.22 & $1 \cdot 1$ & 0.1 & 1.0 & 0.2 & -0.09 & 0.17 \\
\hline Fasting glucose $(\mathrm{mmol} / \mathrm{l})$ & $5 \cdot 4$ & 0.6 & $5 \cdot 4$ & 0.5 & -0.04 & 0.60 & $5 \cdot 5$ & 0.5 & $5 \cdot 4$ & 0.5 & -0.15 & 0.52 \\
\hline Fasting insulin $(\mathrm{mU} / \mathrm{ml})$ & $7 \cdot 7$ & 3.3 & 7.9 & 4.0 & 0.20 & 3.68 & 8.6 & 3.9 & 7.9 & 3.8 & -0.66 & 2.44 \\
\hline \multicolumn{13}{|c|}{ Fatty acids in cholesteryl esters ( $\mathrm{g} / 100 \mathrm{~g}$ total fatty acids) } \\
\hline Total SFA & 11.6 & 1.2 & 14.5 & 1.2 & $2 \cdot 9$ & $1 \cdot 1$ & $13 \cdot 2$ & 1.9 & 11.2 & 0.9 & $-2 \cdot 0^{*}$ & 1.9 \\
\hline Total MUFA & $27 \cdot 1$ & $2 \cdot 1$ & 29.4 & $3 \cdot 1$ & $2 \cdot 3$ & $2 \cdot 3$ & $28 \cdot 1$ & 3.1 & $27 \cdot \overline{5}$ & $2 \cdot 0$ & $-0 \cdot 6^{*}$ & $2 \cdot 2$ \\
\hline Oleic acid $(18: 1 n-9)$ & $22 \cdot 8$ & 1.9 & $23 \cdot 3$ & 1.5 & 0.5 & $2 \cdot 1$ & 22.5 & 1.8 & 23.6 & 1.6 & $1 \cdot 1$ & 1.9 \\
\hline Total $n-6$ PUFA & 58.7 & 3.5 & 52.4 & 3.4 & $-6 \cdot 3$ & $2 \cdot 3$ & $55 \cdot 2$ & 4.7 & 58.9 & 3.1 & $3.7^{*}$ & 3.6 \\
\hline Linoleic acid $(18: 2 n-6)$ & $49 \cdot 0$ & 4.4 & $43 \cdot 1$ & 4.0 & -5.9 & $2 \cdot 4$ & $45 \cdot \overline{5}$ & $4 \cdot 3$ & 48.5 & $4 \cdot 3$ & $3 \cdot 1^{*}$ & 3.2 \\
\hline Total $n$-3 PUFA & $2 \cdot 2$ & 0.8 & $3 \cdot 1$ & 0.6 & 0.9 & 0.4 & 3.0 & 0.6 & $2 \cdot 1$ & 0.8 & $-0.9^{*}$ & 0.4 \\
\hline
\end{tabular}

SFA, saturated fatty acid; MUFA, monounsaturated fatty acid; PUFA, polyunsaturated fatty acid.

Mean values were significantly different from those of the SFA-rich diet $\left(2 \times 2\right.$ crossover study design, ANOVA; $\left.{ }^{*} \leq 0.05\right)$.

Mean value had a trend towards a significant difference from that of the SFA-rich diet $(2 \times 2$ crossover study design, ANOVA; $\dagger P=0.07)$.

Observed resting and postprandial substrate oxidation rates at the end of the two diets did not differ from those observed with the same test meal before the diets (Fig. 2). The observed difference between SFA- and MFA-rich test meals given at the end of the diets, however, appeared smaller than observed previously (Piers et al. 2002). This was not unexpected, as the enlarged fat store at the end of the SFA-rich diet possibly promoted a chronic increase in fat oxidation unrelated to the meal consumed (Flatt, 1995). This chronic increase in fat oxidation rate, in response to a high fat intake, eventually permits a new plateau of fat balance and energy balance to be achieved at a higher body weight and FM; this is why body weight and FM do not keep increasing linearly (Flatt, 1995). While there is now considerable evidence in human subjects that dietary fat type can influence fat oxidation rates (Jones et al. 1985, 1992; Jones \& Schoeller, 1988; DeLany et al. 2000; Piers et al. 2002), until the present study there has been no information on the impact this may have on body weight and body composition. However, animal studies indicate the potential differential impact of different dietary fats on body composition in the longer term (Matsuo et al. 1995; Takeuchi et al. 1995; Bell et al. 1997). Bell et al. (1997) compared MUFA-rich (rapeseed oil) and SFA-rich (beef fat) diets (40.8\% energy from fat) in mice. Animals fed the MUFA-rich diet deposited significantly less body fat than mice fed the high-SFA diet: entirely consistent with our present results in human subjects.

Throughout our present study, foods and beverages were supplied as modules of fixed macronutrient composition, but differing energy content, as described by Stubbs et al. (1995). This procedure was adopted to determine if subjects would then consume more food on the SFA-rich diet. In fact, taste and satiety responses to the two diets did not differ and they allowed very similar total energy and total fat intake (Table 2). Hence, change in the type of fat per se did not appear to affect total fat consumption. The observed significant sequence effect on fibre intake cannot be explained. Although all modules were designed to have similar fibre content, subject selection of individual modules resulted in a difference of $5 \mathrm{~g} / \mathrm{d}$ between diets in fibre intake (Table 2). This difference, however, does not account for the differences in the change in FM, as this difference persisted after statistical adjustment for fibre intake. There were large differences between diets in the type of fat consumed. The difference in SFA intake between diets was $48 \mathrm{~g} / \mathrm{d}$ (Table 2). These differences in fat intake were associated with blood lipid changes that provide objective evidence of dietary adherence (Table 4). On the SFA-rich diet, the plasma SFA fraction of plasma CE was $4.9 \%$ higher $(P=0.001)$, while total $n-6$ PUFA was $10.3 \%$ lower $(P=0.0001)$. In particular, the proportion of linoleic acid $(18: 2 n-6)$, an essential fatty acid, fell markedly on the SFA-rich diet and rose on the MUFA-rich diet, a difference of $-9.0 \%(P=0.001)$. The concentration of linoleic acid in plasma CE is well known to be reflective of dietary linoleic acid intake (Sinclair et al. 1987; Sanders et al. 1994; Sarkkinen et al. 1994). The difference in the change in oleic acid $(18: 1 n-9)$ was not significant $(P=0.61)$, but oleic acid is a non-essential fatty acid that can be synthesised from carbohydrate and is a poor indicator of dietary oleic acid intake (Sanders et al. 1994). Changes in the lipid profile (Table 4) were also consistent with dietary change (Table 2), with total and LDL-cholesterol rising on the SFA-rich diet and falling on the MUFA-rich diet, in accordance with the well established effects of SFA and MUFA (Hegsted et al. 1993). We did not include a washout period between the two diet periods, because previous studies have indicated that this is unnecessary when the dietary intervention period is $>3$ weeks (Bonanome \& Grundy, 1988; Fuentes et al. 2001).

There are several limitations in the present study. The first is the small number of subjects who participated. 
The results need confirmation in larger studies of a similar design. Second, it is possible that subjects consciously restricted their intake on the MUFA-rich diet, as they were free-living and their meals were not supervised. Subjects had no reason to do this, however, as they were never told that the purpose of the study concerned changes in body weight. Throughout the study, they were constantly encouraged to eat to appetite and extra food modules were always made available. From their expressed preference for the MUFA-rich diet, we would possibly have expected more overeating in this phase, rather than the SFA-rich phase of the study. Third, it is possible that subjects were more physically active during the MUFA-rich diet. There was some subjective evidence for this from the questionnaire. Whether increased physical activity was the cause, or consequence, of the loss of FM on the MUFA-rich diet cannot be determined from the present study. Although it remains possible theoretically that the observed differences are the result of a pure coincidence, this seems unlikely given that all subjects systematically gained weight and FM on the SFA-rich diet and all subjects systematically lost weight and FM on the MUFA-rich diet.

The results of our current study are consistent with some recent findings in other dietary studies. An ad libitum diet low in animal fat and refined carbohydrates and rich in low-glycaemic-index foods, MUFA, $n-3$ PUFA and phyto-oestrogens favourably modified the hormonal profile of postmenopausal women when compared with a highSFA control diet (Berrino et al. 2001). That MUFA-rich diet also significantly decreased body weight (4.06 v. $0.54 \mathrm{~kg}$ in the control group), waist:hip ratio, total cholesterol, fasting glucose level and area under the insulin curve after an oral glucose tolerance test. In another recent study (McManus et al. 2001), a moderate fat (35\% energy), controlled energy, Mediterranean-style diet rich in MUFA (15-20\% energy) has been shown to be superior to a low-fat ( $20 \%$ energy) high-carbohydrate diet for weight reduction. In that study, there was superior long-term participation and adherence with the MUFA-rich diet and greater weight loss. Other studies comparing SFArich to MUFA-rich diets, however, have not reported significant differences in the magnitude of weight loss (Mata et al. 1992; deLorgeril et al. 1999; Williams et al. 1999; Vessby et al. 2001). The mechanisms whereby SFA become more readily diverted to adipose tissue storage and the effects this may then have on body metabolism and energy balance remain to be elucidated. The peroxisome proliferator-activated receptors $(\alpha, \delta$ (or $\beta$ ) and $\gamma$ ), a subfamily of the nuclear receptor gene family, have emerged recently as important physiological sensors of lipid levels and may provide a molecular mechanism whereby dietary fatty acids modulate lipid homeostasis. In vitro studies show that unsaturated fatty acids (both MUFA and/or PUFA) are more effective in stimulating peroxisome proliferator-activated receptor $\alpha$ than SFA (Kliewer et al. 1997; Halvorsen et al. 2001). Such results suggest a plausible mechanism, among others, for our present observations.

In conclusion, the results of our present study suggest that the previously observed greater fat oxidation rate following a MUFA-rich test meal, as compared with a
SFA-rich test meal, can result in a small but significant reduction in body weight and FM in men whose dietary SFA content is largely replaced with MUFA, within 4 weeks. This effect could possibly have been enhanced by subtle, unconscious, increases in physical activity and/or decreases in energy intake; however, there were no detectable changes in basal or postprandial EE. This is a novel result and it requires confirmation, perhaps using a whole-body calorimeter to measure EE and substrate oxidation rates in a larger number of subjects over a longer period of time, possibly in conjunction with the doubly-labelled water technique to measure free-living EE.

\section{Acknowledgements}

This project was supported by a project grant (no. 981019) from the National Health and Medical Research Council, Australia. We would like to thank the volunteers who participated in this study, without whose cooperation none of this would be possible. We would also like to thank: Ms Margaret Halley and Ms Cheryl Greig for help with the preparation of the diets; $\mathrm{Mr}$ David Hamilton-Smith and the Food Service Department, The Kingston Centre, Cheltenham, Victoria, Australia, for the donated provisions and food preparation facilities; Ms Elaine Yeow for logistical support; Ms Connie Karschimkus for processing the blood and urine samples; Dr Boyd Strauss for use of the body composition facility; Mr Mark Neumann for the serum fatty acid analyses; Dr Zhiqiang Wang and Dr Bircan Erbas for statistical advice.

\section{References}

Bell RR, Spencer MJ \& Sherriff JL (1997) Voluntary exercise and monounsaturated canola oil reduce fat gain in mice fed diets high in fat. J Nutr 127, 2006-2010.

Ben-Porat M, Sideman S \& Bursztein S (1983) Energy metabolism rate equation for fasting and postabsorptive subjects. Am $J$ Physiol 244, R764-R769.

Berrino F, Bellati C, Secreto G, et al. (2001) Reducing bioavailable sex hormones through a comprehensive change in diet: the diet and androgens (DIANA) randomized trial. Cancer Epidemiol Biomarkers Prev 10, 25-33.

Bligh EG \& Dyer WJ (1959) A rapid method of total lipid extraction and purification. Can J Biochem Pharmacol 37, 911-917.

Blundell JE \& MacDiarmid JI (1997) Fat as a risk factor for overconsumption: satiation, satiety, and patterns of eating. $J \mathrm{Am}$ Diet Assoc 97, S63-S69.

Bonanome A \& Grundy SM (1988) Effect of dietary stearic acid on plasma cholesterol and lipoprotein levels. $N$ Engl J Med 318, 1244-1248.

Bray GA \& Popkin BM (1998) Dietary fat intake does affect obesity! Am J Clin Nutr 68, 1157-1173.

Callaway CW, Chumlea WC, Bouchard C, et al. (1988) In Anthropometric Standardisation Reference Manual, pp. 39-54 [TG Lohman, AF Roche and R Martorell, editors]. Champaign, IL: Human Kinetics Books.

DeLany JP, Windhauser MM, Champagne CM \& Bray GA (2000) Differential oxidation of individual dietary fatty acids in humans. Am J Clin Nutr 72, 905-911. 
deLorgeril M \& Salen P (2000) Modified Cretan Mediterranean diet in the prevention of coronary heart disease and cancer. World Rev Nutr Diet 87, 1-23.

deLorgeril M, Salen P, Martin JL, Monjaud I, Delaye J \& Mamelle N (1999) Mediterranean diet traditional risk factors, and the rate of cardiovascular complications after myocardial infarction: final report of the Lyon Diet Heart Study. Circulation 99, 779-785.

Elia M \& Livesey G (1988) Theory and validity of indirect calorimetry during net lipid synthesis. Am J Clin Nutr 47, 591-607.

English R \& Lewis J (1991) Nutritional Values of Australian Foods. Canberra: Australian Government Printing Service.

Flatt JP (1995) Use and storage of carbohydrate and fat. Am J Clin Nutr 61, 952S-959S.

Food and Agriculture Organization/World Health Organization/ United Nations University (1985) Energy and Protein Requirements: Report of a Joint FAO/WHO/UNU Expert Consultation. Technical Report Series no. 724, Geneva: WHO.

Fuentes F, Sanchez E, Sanchez F, et al. (2001) Mediterranean and low-fat diets improve endothelial function in hypercholesterolemic men. Ann Intern Med 134, 1115-1119.

Garg A (1994) High-monounsaturated fat diet for diabetic patients. Is it time to change the current dietary recommendations? Diabetes Care 17, 242-246.

Halvorsen B, Rustan AC, Madsen L, et al. (2001) Effects of longchain monounsaturated and $n-3$ fatty acids on fatty acid oxidation and lipid composition in rats. Ann Nutr Metab $\mathbf{4 5}$, $30-37$.

Hegsted DM, Ausman LM, Johnson JA \& Dallal GE (1993) Dietary fat and serum lipids: an evaluation of the experimental data. Am J Clin Nutr 57, 875-883.

Jones PJ, Pencharz PB \& Clandinin MT (1985) Whole body oxidation of dietary fatty acids: implications for energy utilization. Am J Clin Nutr 42, 769-777.

Jones PJ, Ridgen JE, Phang PT \& Birmingham CL (1992) Influence of dietary fat polyunsaturated to saturated ratio on energy substrate utilization in obesity. Metab Clin Exp 41 , 396-401.

Jones PJ \& Schoeller DA (1988) Polyunsaturated:saturated ratio of diet fat influences energy substrate utilization in the human. Metab Clin Exp 37, 145-151.

Kliewer SA, Sundseth SS, Jones SA, et al. (1997) Fatty acids and eicosanoids regulate gene expression through direct interactions with peroxisome proliferator-activated receptors alpha and gamma. Proc Natl Acad Sci USA 94, 4318-4323.

Lissner L \& Heitmann BL (1995) Dietary fat and obesity: evidence from epidemiology. Eur J Clin Nutr 49, 79-90.

Livesey G \& Elia M (1988) Estimation of energy expenditure, net carbohydrate utilization, and net fat oxidation and synthesis by indirect calorimetry: evaluation of errors with special reference to the detailed composition of fuels. Am J Clin Nutr 47, $608-628$.

McCarron DA \& Reusser ME (1996) Body weight and blood pressure regulation. Am J Clin Nutr 63, 423S-425S.

McManus K, Antinoro L \& Sacks F (2001) A randomized controlled trial of a moderate-fat, low-energy diet compared with a low fat, low-energy diet for weight loss in overweight adults. Int J Obes Relat Metab Disord 25, 1503-1511.

Mata P, Garrido JA, Ordovas JM, et al. (1992) Effect of dietary monounsaturated fatty acids on plasma lipoproteins and apolipoproteins in women. Am J Clin Nutr 56, 77-83.
Matsuo T, Shimomura Y, Saitoh S, Tokuyama K, Takeuchi H \& Suzuki M (1995) Sympathetic activity is lower in rats fed a beef tallow diet than in rats fed a safflower oil diet. Metab Clin Exp 44, 934-939.

Piers LS, Walker KZ, Stoney RM, Soares MJ \& O’Dea K (2002) The influence of the type of dietary fat on postprandial fat oxidation rates: monounsaturated (olive oil) versus saturated fat (cream). Int J Obes Relat Metab Disord 26, 814-821.

Raison JM, Achimastos AM \& Safar ME (1992) Sex-dependence of body fat distribution in patients with obesity and hypertension. Clin Exper Hypertens 14A, 505-525.

Sanders K, Johnson L, O'Dea K \& Sinclair AJ (1994) The effect of dietary fat level and quality on plasma lipoprotein lipids and plasma fatty acids in normocholesterolemic subjects. Lipids $\mathbf{2 9}$, 129-138.

Sarkkinen ES, Agren JJ, Ahola I, Ovaskainen ML \& Uusitupa MI (1994) Fatty acid composition of serum cholesterol esters, and erythrocyte and platelet membranes as indicators of long-term adherence to fat-modified diets. Am J Clin Nutr 59, 364-370.

Sinclair AJ, O’Dea K, Dunstan G, Ireland PD \& Niall M (1987) Effects on plasma lipids and fatty acid composition of very low fat diets enriched with fish or kangaroo meat. Lipids 22, 523-529.

Storlien LH, Hulbert AJ \& Else PL (1998) Polyunsaturated fatty acids membrane function and metabolic diseases such as diabetes and obesity. Curr Opin Clin Nutr Metab Care 1, 559-563.

Storlien LH, Tapsell LC, Fraser A, et al. (2001) Insulin resistance. Influence of diet and physical activity. World Review Nutr Diet 90, 26-43.

Stubbs RJ (1998) Nutrition Society Medal Lecture. Appetite, feeding behaviour and energy balance in human subjects. Proc Nutr Soc 57, 341-356.

Stubbs RJ, Harbron CG, Murgatroyd PR \& Prentice AM (1995) Covert manipulation of dietary fat and energy density: effect on substrate flux and food intake in men eating ad libitum. Am J Clin Nutr 62, 316-329.

Takeuchi H, Matsuo T, Tokuyama K, Shimomura Y \& Suzuki M (1995) Diet-induced thermogenesis is lower in rats fed a lard diet than in those fed a high oleic acid safflower oil diet, a safflower oil diet or a linseed oil diet. J Nutr 125, 920-925.

Vessby B, Unsitupa M, Hermansen K, et al. (2001) Substituting dietary saturated for monounsaturated fat impairs insulin sensitivity in healthy men and women: The KANWU Study. Diabetologia 44, 312-319.

Walker KZ, O'Dea K, Johnson L, et al. (1996) Body fat distribution and non-insulin-dependent diabetes: comparison of a fiber-rich, high-carbohydrate, low-fat $(23 \%)$ diet and a $35 \%$ fat diet high in monounsaturated fat. Am J Clin Nutr 63, $254-260$.

Walker KZ, O’Dea K, Nicholson GC \& Muir JG (1995) Dietary composition, body weight, and NIDDM. Comparison of highfiber, high-carbohydrate, and modified-fat diets. Diabetes Care 18, 401-403.

Willet WC (2002) Dietary fat plays a major role in obesity: no. Obes Rev 3, 59-68.

Williams CM, Francis-Knapper JA, Webb D, et al. (1999) Cholesterol reduction using manufactured foods high in monounsaturated fatty acids: a randomized crossover study. $\mathrm{Br} J$ Nutr 81, 439-446.

Yandell BS (1997) Practical Data Analysis for Designed Experiments. Boca Raton, FL: CRC Press. 